PROCEEDINGS OF THE

AMERICAN MATHEMATICAL SOCIETY

Volume 129, Number 7 , Pages 2085-2092

S 0002-9939(00)05843-3

Article electronically published on December 28, 2000

\title{
SIMILARITY TO A CONTRACTION AND HYPERCONTRACTIVITY OF COMPOSITION OPERATORS
}

\author{
NIZAR JAOUA
}

(Communicated by David R. Larson)

\begin{abstract}
On the Hardy spaces $H^{p}$ with $1 \leq p<\infty$, we consider the composition operators induced by analytic self-maps of the open unit disc $D$. First, we characterize those which are similar to contractions. Then, we give some necessary and sufficient conditions for them to be hypercontractive. Finally, we prove that, among those ones, only the zero-symbol composition operator sends $H^{p}$ into $H^{\infty}$ with a norm less than or equal to 1 .
\end{abstract}

\section{INTRODUCTION}

Throughout this paper, we denote by $D$ the open unit disc in the complex plane, by $H(D)$ the space of holomorphic functions on $D$ and by $H(D, D)$ the subset of $H(D)$ consisting of all self-maps of $D$. We also denote by $\mathbb{N}^{*}$ the set of integers larger than one : $\mathbb{N}^{*}=\{1,2, \cdots\}$.

Let $\varphi$ be in $H(D, D)$. On appropriate subspaces of $H(D)$, the composition operator induced by the symbol $\varphi$ is defined by $C_{\varphi} f:=f \circ \varphi$. We recall that the Hardy space $H^{p}(0<p<\infty)$ is the subspace of $H(D)$ consisting of all functions satisfying

$$
\|f\|_{p}:=\left(\sup _{0 \leq r<1} \frac{1}{2 \pi} \int_{0}^{2 \pi}\left|f\left(r e^{i \theta}\right)\right|^{p} d \theta\right)^{\frac{1}{p}}<\infty .
$$

For $p \geq 1$, this gives a norm for which $H^{p}$ is a Banach space. We also recall that the space $H^{\infty}$, endowed with the norm $\|f\|_{\infty}:=\sup _{z \in D}|f(z)|$, is a Banach space too. From these definitions, we get the following proper inclusions:

$$
H^{\infty} \subset H^{\beta p} \subset H^{p} \quad(0<p<\infty, 1<\beta<\infty) .
$$

It is well known that $C_{\varphi}$ is continuous on $H^{p}(0<p<\infty)$ (see [10] and [14]) and that, for $p \geq 1, C_{\varphi}$ is a contraction (i.e. $\left\|C_{\varphi}\right\| \leq 1$ ) if and only if $\varphi(0)=0$ (see Theorem 2.1).

Here, we will characterize a subclass of composition operators that contains each $C_{\varphi}$ which is similar to a contraction. In Section 3, we show that the existence of a fixed point in $D$, for the symbol $\varphi$, is a necessary and sufficient condition for $C_{\varphi}$ to

Received by the editors March 10, 1999 and, in revised form, November 17, 1999.

1991 Mathematics Subject Classification. Primary 47B38, 47B65.

Key words and phrases. Composition operators, Hardy spaces, contraction, hypercontractive (or $\beta$-contractive) operator. 
be in that class. In particular, we observe that if $C_{\varphi}$ is polynomially bounded, then it is similar to a contraction. G. Pisier has shown that this is not true for every operator on a Hilbert space (see 13] for details). In the case where the contraction is isometric and the symbol is analytic on a neighborhood of $\bar{D}$, we give a more precise characterization.

In Section 4, we study the class of all $C_{\varphi}$ 's sending $H^{p}$ into $H^{\beta p}(\beta>1)$ with a norm less than or equal to 1 . The qualitative aspect of this problem (i.e. sending $H^{p}$ into $H^{\beta p}$ ) has been solved by H. Hunziker and H. Jarchow ([7]). For the quantitative aspect (i.e. sending $H^{p}$ into $H^{\beta p}$ with norm $\leq 1$ ), we give some necessary and sufficient conditions. $C_{\varphi}$ 's satisfying the second aspect are said to be hypercontractive or also $\beta$-contractive. At the end of Section 4 , we show that, among those ones, only the zero-symbol composition operator is a contraction from $H^{p}$ into $H^{\infty}$.

Section 2 is devoted to some results focused on the symbols $\varphi$ and on the operators $C_{\varphi}$ sending $H^{p}$ into $H^{\beta p}(1 \leq \beta, p<\infty)$.

\section{Preliminaries}

It is well known (cf. [5]) that, for each $f \in H^{p}, f^{*}\left(e^{i \theta}\right):=\lim _{r \rightarrow 1} f\left(r e^{i \theta}\right)$ exists almost everywhere on the unit circle $\partial D$. A function $\varphi$ in $H(D, D)$ is said to be inner if $\left|\varphi^{*}\right|=1$ almost everywhere.

Theorem 2.1 (cf. 2], p. 123). For all $\varphi \in H(D, D)$ and $p \in\left[1,+\infty\left[, C_{\varphi}\right.\right.$ is bounded on $H^{p}$ and we have

$$
\sup _{|z|<1}\left(\frac{1-|z|^{2}}{1-|\varphi(z)|^{2}}\right)^{\frac{1}{p}} \leq\left\|C_{\varphi}\right\| \leq\left(\frac{1+|\varphi(0)|}{1-|\varphi(0)|}\right)^{\frac{1}{p}} .
$$

Moreover, $C_{\varphi}$ is an isometry if and only if $\varphi$ is inner and vanishes at 0.

Let $X$ be a Banach space. We recall that $T: X \rightarrow X$ is an isometry if $\|T x\|=\|x\|$ for all $x \in X$. The following well-known proposition (see 1], p. 213) describes the spectrum $\sigma(T)$ of such an operator. For sake of completeness, we give an elementary proof.

Proposition 2.2. If $T: X \rightarrow X$ is an isometry, then either $\sigma(T) \subseteq \partial D$ or $\sigma(T)=\bar{D}$.

Proof. If $T$ is onto, $\sigma(T) \subseteq \partial D$; otherwise $E:=\sigma(T) \cap D$ is a closed subset of $D$ containing 0. Let $F=D-E=\sigma(T)^{c} \cap D$ be the complement of $E$ in $D$. If $\lambda \in D$, $\lambda_{n} \in F$ and $\lambda_{n} \rightarrow \lambda$, then for all $x \in X$, we have

$$
\left\|\left(T-\lambda_{n} I\right) x\right\| \geq\|T x\|-\left|\lambda_{n}\right|\|x\|=\left(1-\left|\lambda_{n}\right|\right)\|x\| \geq \frac{1-|\lambda|}{2}\|x\|=\delta\|x\|
$$

for large $n$, which implies that $\left.\| T-\lambda_{n} I\right)^{-1} \| \leq \delta^{-1}$. And this implies (cf. [4]) that $\lambda \in \sigma(T)^{c}$. Therefore, $F$ is equally closed in $D$. The connectedness of $D$ implies that $E=D$, and $\sigma(T)=\bar{D}$.

Remark. Proposition 2.2 can be seen as a consequence of the fact that $\partial \sigma(T)$ is contained in $\sigma_{a p}(T)$ (Proposition 6.7 in [1]). This fact implies that, for any $\lambda \in$ $\partial \sigma(T)$, there is a sequence $\left(x_{n}\right)_{n}$ with $\left\|x_{n}\right\|=1$ such that $\left\|(T-\lambda I) x_{n}\right\| \rightarrow 0$. Since $T$ is an isometry, it is easy to see that $|\lambda|=1$, and the result follows by connectedness of $D$. 
The following proposition can be proved by using the factorization theorem of F. Riesz ([5], p. 20). It justifies the reason for which one can study hypercontractivity only on the Hilbert space $H^{2}$. If it sends $H^{p}$ into $H^{\beta p}$, for some $1<\beta<\infty$, $C_{\varphi}$ is said to be $\beta$-bounded, and we denote its norm by $\left\|C_{\varphi}\right\|_{p, \beta p}$.

Proposition 2.3. If $C_{\varphi}$ is hypercontractive on $H^{p}$ for some $1 \leq p<\infty$, then the same is true for all $1 \leq p<\infty$.

In the next theorem, $\left(\varphi_{n}\right)_{n \in \mathbb{N}}$ denotes the iterate sequence of the map $\varphi$. The uniform convergence on every compact set of $D$ is denoted by $\stackrel{u . c}{\longrightarrow}$.

Theorem 2.4 (cf. [3] or [16]). If $\varphi \in H(D, D)$ has no fixed point in $D$, then there exists a unique point $\zeta \in \partial D$ (called the Denjoy-Wolff point of $\varphi$ ) such that $\varphi_{n} \stackrel{u . c}{\rightarrow} \zeta$.

\section{Similarity to A CONTRACtion}

Let $X$ be a Banach space. $T: X \rightarrow X$ is said to be polynomially bounded if there exists $M>0$ such that $\|P(T)\| \leq M\|P\|_{\infty}$ for every polynomial $P$, where $\|P\|_{\infty}=\sup \{|P(z)| ;|z| \leq 1\}$.

Theorem 3.1. In the following assertions, (1), (3) and (4) are equivalent for all $1 \leq p<\infty$. In particular, for $p=2$, they are equivalent to (2).

(1) $C_{\varphi}$ is similar to a contraction.

(2) $C_{\varphi}$ is polynomially bounded.

(3) $C_{\varphi}$ is power bounded.

(4) $\varphi$ has a fixed point in D.

Proof. (1) $\Longrightarrow(2)$ By hypothesis, there exists an invertible operator $S$ on $H^{2}$ such that

$$
\begin{aligned}
C_{\varphi} & =S^{-1} C S \quad \text { where } C \text { is a contraction on } H^{2} . \\
P\left(C_{\varphi}\right) & =S^{-1} P(C) S . \\
\left\|P\left(C_{\varphi}\right)\right\| & \leq\left\|S^{-1}\right\|\|P(C)\|\|S\| \leq\left\|S^{-1}\right\|\|S\|\|P\|_{\infty} .
\end{aligned}
$$

The last estimate follows from the famous von Neumann's inequality.

$(2) \Longrightarrow(3)$ This is immediate.

$(3) \Longrightarrow(4)$ Assume that $\varphi$ has no fixed point in $D$. By the weak form of the Denjoy-Wolff theorem, we have $\left|\varphi_{n}\right| \stackrel{u . c}{\rightarrow} 1$. Since

$$
\left\|C_{\varphi}^{n}\right\|=\left\|C_{\varphi_{n}}\right\| \geq\left(1-\left|\varphi_{n}(0)\right|^{2}\right)^{-\frac{1}{p}}
$$

we conclude that

$$
\lim _{n \rightarrow \infty}\left\|C_{\varphi}^{n}\right\|=\infty
$$

This is in contradiction with (3).

$(4) \Longrightarrow(1)$ Let $a \in D$ be a fixed point of $\varphi$. Set

$$
\psi=\varphi_{a} \circ \varphi \circ \varphi_{a} \quad \text { where } \quad \varphi_{a}(z)=\frac{a-z}{1-\bar{a} z} .
$$

$\varphi_{a}$ is a holomorphic automorphism of $D$ and $\varphi_{a}^{-1}=\varphi_{a}$. Since $\psi \in H(D, D)$ and $\psi(0)=0$, by Theorem [2.1] $C_{\psi}$ is a contraction on $H^{p}$. Now, the identity $\varphi=\varphi_{a} \circ \psi \circ \varphi_{a}^{-1}$ implies that $C_{\varphi}=C_{\varphi_{a}}^{-1} C_{\psi} C_{\varphi_{a}}$, and this completes the proof. 
E. Nordgren ([10]) has shown that if $C_{\varphi}^{n}$ is compact for some $n \in \mathbb{N}^{*}$, then $\varphi$ has a fixed point in $D$. As a consequence of this result and Theorem [3.1, we have the following.

Corollary 3.2. If $C_{\varphi}^{n}$ is compact for some $n \in \mathbb{N}^{*}$, then $C_{\varphi}$ is similar to a contraction.

In order to study the similarity with an isometry on $H^{p}$, we need the following theorem (cf. [9]) which provides the spectrum of $C_{\varphi}$ in a special case.

Theorem 3.3. Suppose $\varphi$ is analytic on a neighborhood of $\bar{D}$, not inner and with a fixed point a in D. If $C_{\varphi}^{n}$ is compact on $H^{p}(1 \leq p<\infty)$ for no $n \in \mathbb{N}^{*}$, then there exists $0<\rho<1$ such that

$$
\sigma\left(C_{\varphi}\right)=\{\lambda \in \mathbb{C} ;|\lambda| \leq \rho\} \cup\left\{\left(\varphi^{\prime}(a)\right)^{n} ; n \in \mathbb{N}^{*}\right\} \cup\{1\} .
$$

Theorem 3.4. Suppose $\varphi$ is analytic on a neighbourhood of $\bar{D}$. Then the following are equivalent:

(1) $C_{\varphi}$ is similar to an isometry.

(2) $\varphi$ is inner and has a fixed point in $D$.

Proof of Theorem 3.4 (1) $\Longrightarrow(2)$ By Theorem 3.1, $\varphi$ has necessarily a fixed point in $D$. Now, assume that $\varphi$ is not inner. Since $C_{\varphi}^{n}$ is compact for no $n \in \mathbb{N}^{*}$ (an operator which is similar to an isometry cannot be compact in infinite dimension), by Theorem 3.3, $\sigma\left(C_{\varphi}\right) \neq \bar{D}$ and $\sigma\left(C_{\varphi}\right) \not \subset \partial D$. This deduction together with Theorem 2.2 leads to a contradiction with (1). Consequently, $\varphi$ is necessarily inner.

$(2) \Longrightarrow(1)$ This can be proved in the same way as $(4) \Longrightarrow(1)$ of Theorem 3.1 Note that, here, $C_{\psi}$ is an isometry since $\psi$ is inner and vanishes at 0 (see Theorem 2.1).

Question. Theorem 3.3 provides the spectrum of $C_{\varphi}$ with $\varphi$ analytic on a neighborhood of $\bar{D}$. Is this condition on $\varphi$ necessary in Theorem 3.4 ?

The next corollary follows immediately from Theorem 3.4 and a known result saying that $C_{\varphi}$ is invertible on $H^{p}$ if and only if $\varphi$ is an automorphism of $D$ (see [11] or [2] or more recently [6]).

Corollary 3.5. A composition operator is similar to an isometric isomorphism on $H^{p}$ if and only if its symbol is an elliptic automorphism of D.

\section{Hypercontractivity}

F. B. Weissler ([15]) has characterized the hypercontractivity of convolution operators by Poisson kernels $P_{r}(\theta):=\frac{1-r^{2}}{1+r^{2}-2 r \cos \theta}(0<r<1)$. Those operators are defined on $L^{p}(\partial D, m)$ by : $\left(\left[P_{r}\right] f\right)\left(e^{i \theta}\right)=\sum_{-\infty}^{+\infty} \hat{f}(n) r^{|n|} e^{i n \theta}$. He has shown that $\left\|\left[P_{r}\right]\right\|_{L^{p} \rightarrow L^{q}}=1$ (resp., $\left\|\left[P_{r}\right]\right\|_{H^{p} \rightarrow H^{q}}=1$ ) if and only if $r^{2} \leq \frac{p-1}{q-1}$ (resp., $r^{2} \leq \frac{p}{q}$ ) for all $p, q$ such that $1<p<q<\infty$.

This result and the following lemma will allow us to characterize the hypercontractive $C_{\alpha z}$ with $|\alpha| \leq 1$. Note that the symbols $\varphi(z)=\alpha z$ are exactly those for which $C_{\varphi}: H^{2} \rightarrow H^{2}$ is normal (see [11).

Lemma 4.1. Let $f \in H^{\infty}$ with $f(0)=0$ and $\left.q \in\right] 0,+\infty[$. For all $\varepsilon>0$ small enough, we have

$$
\|1+\varepsilon f\|_{q}=1+\frac{q}{4}\|f\|_{2}^{2} \varepsilon^{2}+o\left(\varepsilon^{2}\right)
$$


Proof. For all $z \in D$, one has

$$
(1+\varepsilon f(z))^{\frac{q}{2}}=1+\varepsilon \frac{q}{2} f(z)+\varepsilon^{2} \frac{q}{4}\left(\frac{q}{2}-1\right) f^{2}(z)+o\left(\varepsilon^{2}\right) .
$$

Here, $o\left(\varepsilon^{2}\right)$ does not depend on $z$ since $f$ is bounded. So, we obtain

$$
\begin{aligned}
|1+\varepsilon f(z)|^{q} & =\left|(1+\varepsilon f(z))^{\frac{q}{2}}\right|^{2}=(1+\varepsilon f(z))^{\frac{q}{2}} \overline{(1+\varepsilon f(z))^{\frac{q}{2}}} \\
& =1+\varepsilon q \operatorname{Re}(f(z))+\varepsilon^{2} \frac{q}{2}\left(\frac{q}{2}-1\right) \operatorname{Re}\left(f^{2}(z)\right)+\varepsilon^{2} \frac{q^{2}}{4}|f(z)|^{2}+o\left(\varepsilon^{2}\right) .
\end{aligned}
$$

As $\operatorname{Re}(f)$ and $\operatorname{Re}\left(f^{2}\right)$ are harmonic in $D$ and since $f(0)=0$, by integration on $[0,2 \pi]$ with respect to the measure $\frac{d \theta}{2 \pi}$, one gets

$$
\frac{1}{2 \pi} \int_{0}^{2 \pi}\left|1+\varepsilon f\left(e^{i \theta}\right)\right|^{q} d \theta=1+\varepsilon^{2} \frac{q^{2}}{4}\|f\|_{2}^{2}+o\left(\varepsilon^{2}\right) .
$$

This leads to the desired assertion.

Theorem 4.2. Let $\alpha \in \bar{D}$ and $1<\beta<\infty$. We have equivalence between (1) and (2) and between (3) and (4).

(1) $C_{\alpha z}$ is $\beta$-bounded.

(2) $|\alpha|<1$.

(3) $C_{\alpha z}$ is $\beta$-contractive.

(4) $|\alpha| \leq \beta^{-\frac{1}{2}}$.

Proof. (1) $\Longrightarrow(2)$ Suppose that $|\alpha|=1$. That means $\varphi$ is a rotation of $D$, and then $C_{\varphi}$ sends $H^{2}$ onto itself: this is a contradiction with (1).

$(2) \Longrightarrow(1)$ Since $\|\varphi\|_{\infty}=|\alpha|<1$, one has

$$
C_{\varphi}\left(H^{2}\right) \subset H^{\infty} \subset H^{2 \beta} .
$$

Thus, $C_{\varphi}$ is $\beta$-bounded.

(3) $\Longrightarrow(4)$ Let $g$ be the function defined on $D$ by $g(z)=1+\varepsilon z$ with $\varepsilon>0$ small enough. Since $C_{\alpha z} g=1+\varepsilon f$ where $f(z)=\alpha z$ for all $z \in D$, by Lemma 4.1 applied to this $f$ and to $q=2 \beta$, we get

$$
\left\|C_{\varphi} g\right\|_{2 \beta}=1+\frac{\beta|\alpha|^{2}}{2} \varepsilon^{2}+o\left(\varepsilon^{2}\right) .
$$

On the other hand, this same lemma, applied to $f \equiv z$ and $q=2$, gives

$$
\|g\|_{2}=1+\frac{1}{2} \varepsilon^{2}+o\left(\varepsilon^{2}\right) .
$$

Now, by hypothesis, we have $\left\|C_{\varphi} g\right\|_{2 \beta} \leq\|g\|_{2}$. Let us simplify, then take the limit as $\varepsilon \rightarrow 0$ to obtain $|\alpha|^{2} \leq \frac{1}{\beta}$. This implies (4).

$(4) \Longrightarrow(3)$ This is nothing else but another formulation of Weissler's theorem mentioned at the beginning of this section. Indeed, just observe that $\left\|C_{\alpha z}\right\|_{2,2 \beta}=$ $\left\|\left[P_{r}\right]\right\|_{H^{2} \rightarrow H^{2 \beta}}$ with $r=|\alpha|$.

Corollary 4.3. If $\varphi(0)=0$ and $\|\varphi\|_{\infty} \leq \beta^{-\frac{1}{2}}$, then $C_{\varphi}$ is $\beta$-contractive.

Proof. Set $r=\|\varphi\|_{\infty}$ and $\psi=\frac{1}{r} \varphi$. By Schwarz' lemma, one has $|\varphi(z)| \leq r|z|$. Thus, $\psi \in H(D, D)$. On the other hand, the identities $C_{\varphi}=C_{r \psi}=C_{\psi} C_{r z}$ imply that

$$
\left\|C_{\varphi}\right\|_{1, \beta} \leq\left\|C_{\psi}\right\|_{\beta, \beta}\left\|C_{r z}\right\|_{1, \beta} \leq 1
$$

The last estimate comes from the fact that $\psi(0)=0$ and from Theorem 4.2 which one can apply because $r \leq \beta^{-\frac{1}{2}}$. 
In the following theorem, we denote by $H_{0}^{2}$ the subspace of $H^{2}$ consisting of all functions vanishing at 0 .

Theorem 4.4. If $C_{\varphi}$ is $\beta$-contractive, then we have the following:

(1) $\left\|C_{\varphi}\right\|_{B\left(H_{0}^{2}\right)} \leq \beta^{-\frac{1}{2}}$.

(2) $\frac{1}{2 \pi} \int_{0}^{2 \pi}\left|g\left(\varphi\left(e^{i \theta}\right)\right)\right|^{2}\left|\varphi\left(e^{i \theta}\right)\right|^{2} d \theta \leq \frac{1}{\beta}\|g\|_{2}^{2} \quad$ for all $g \in H^{2}$. In particular, $\|\varphi\|_{2} \leq \beta^{-\frac{1}{2}}$.

Proof. (1) First, let us suppose that $f \in H^{\infty}$ with $f(0)=0$. Since the contractivity of $C_{\varphi}$ implies that $\varphi(0)=0$, we get $f \circ \varphi \in H^{\infty}$ and $(f \circ \varphi)(0)=0$. Let $\varepsilon>0$ be small enough. By Lemma 4.1 we obtain

$$
\begin{cases}\|1+\varepsilon f \circ \varphi\|_{2 \beta} & =1+\frac{\beta}{2}\|f \circ \varphi\|_{2}^{2} \varepsilon^{2}+o\left(\varepsilon^{2}\right) \\ \|1+\varepsilon f\|_{2} & =1+\frac{1}{2}\|f\|_{2}^{2} \varepsilon^{2}+o\left(\varepsilon^{2}\right)\end{cases}
$$

Now, by hypothesis, one has

$$
\|1+\varepsilon f \circ \varphi\|_{2 \beta} \leq\|1+\varepsilon f\|_{2} .
$$

Thus, after simplifying and taking the limit as $\varepsilon \rightarrow 0$, one concludes that $\beta\|f \circ \varphi\|_{2}^{2} \leq$ $\|f\|_{2}^{2}$. Hence, $\|f \circ \varphi\|_{2} \leq \beta^{-\frac{1}{2}}\|f\|_{2}$.

Let us suppose now that $f \in H_{0}^{2}$. If we denote by $\left(f_{n}\right)_{n}$ the partial sums of $f$, that is, $f_{n}(z)=\sum_{k=1}^{n} \hat{f}(k) z^{k}$, we have $\left\|f_{n}-f\right\|_{2} \rightarrow 0$. The inequality $\left\|f_{n} \circ \varphi\right\|_{2}^{2} \leq \beta^{-1}\left\|f_{n}\right\|_{2}^{2}$ follows from the previous part of the proof (since $f_{n} \in H_{0}^{\infty}$ ), and the desired result is obtained by passing to the limit in this inequality (since $C_{\varphi}$ is bounded on $H^{2}$ ).

(2) This follows from (1) by using the fact that $z g \in H_{0}^{2}$ for all $g \in H^{2}$.

Remark. So far, we have not been able to discover whether the condition $\|\varphi\|_{\infty}<1$ is necessary for $C_{\varphi}$ to $\beta$-contractive. However, one can show that this is true in the case where $\varphi(0)=0$ and $|\varphi(u)-\varphi(v)| \leq k|u-v|^{\frac{1}{\beta}}$ on $(\partial D)^{2}$ with $0<k<2^{\frac{1}{\beta}-1}$ (see [8]).

H. Hunziker and H. Jarchow ([7]) have characterized $\beta$-boundedness of $C_{\varphi}$ 's for all $1 \leq \beta<\infty$. The following theorem gives a version for the limit case $\beta=\infty$.

Theorem 4.5. The following are equivalent:

(1) $\|\varphi\|_{\infty}<1$.

(2) $C_{\varphi}\left(H^{p}\right) \subset H^{\infty}$ for all $1 \leq p<\infty$.

(3) $C_{\varphi}\left(H^{p}\right) \subset H^{\infty}$ for some $1 \leq p<\infty$.

Proof. (1) $\Longrightarrow(2)$ It is well known (cf. [17]) that the point evaluation operator $\delta_{z}$, defined by $\delta_{z}(f)=f(z)$, is bounded on $H^{p}$ and $\left\|\delta_{z}\right\|=\left(1-|z|^{2}\right)^{-\frac{1}{p}}$. For all $f \in H^{p}$ and $z \in D$, we have

$$
|f(\varphi(z))| \leq\left\|\delta_{\varphi(z)}\right\|_{\left(H^{p}\right)^{*}}\|f\|_{p}=\left(1-|\varphi(z)|^{2}\right)^{-\frac{1}{p}}\|f\|_{p} \leq\left(1-\|\varphi\|_{\infty}^{2}\right)^{-\frac{1}{p}}\|f\|_{p} .
$$

Thus, $f \circ \varphi \in H^{\infty}$ and $\|f \circ \varphi\|_{\infty} \leq\left(1-\|\varphi\|_{\infty}^{2}\right)^{-\frac{1}{p}}\|f\|_{p}$.

$(2) \Longrightarrow(3)$ This is immediate.

$(3) \Longrightarrow(1)$ For every $\zeta \in \partial D$, the function $z \longmapsto(\zeta-z)^{-\frac{1}{2 p}}$ belongs to $H^{p}$. So by hypothesis, there exists $c>1$ such that $|\zeta-\varphi(z)|^{-\frac{1}{2 p}} \leq c$. In other words, there 
exists $\delta \in] 0,1[$ such that

$$
|\zeta-\varphi(z)| \geq \delta \quad \text { for all } z \in D .
$$

Let $z$ be in $D$ such that $\varphi(z) \neq 0$. By $(*)$, applied to $\zeta=\frac{\varphi(z)}{|\varphi(z)|}$, we obtain

$$
1-|\varphi(z)|=|\varphi(z)|\left|\frac{1}{|\varphi(z)|}-1\right|=\left|\varphi(z)\left(\frac{1}{|\varphi(z)|}-1\right)\right| \geq \delta .
$$

Thus, $|\varphi(z)| \leq 1-\delta$; $z$ being arbitrary, we conclude that $\|\varphi\|_{\infty} \leq 1-\delta<1$.

We end this paper with the following theorem which says that $C_{0}$ is the only contractive composition operator from $H^{p}(1 \leq p<\infty)$ into $H^{\infty}$.

Theorem 4.6. The following are equivalent:

(1) $C_{\varphi}: H^{p} \rightarrow H^{\infty}$ is a contraction.

(2) $\varphi \equiv 0$.

Proof. (1) $\Longrightarrow(2)$ For all $z \in D$ and $f \in H^{p}$ with $\|f\|_{p}=1$, one has $|f(\varphi(z))| \leq$ $\|f \circ \varphi\|_{\infty} \leq 1$. Thus, $\left\|\delta_{\varphi(z)}\right\|_{\left(H^{p}\right)^{*}}=\left(1-|\varphi(z)|^{2}\right)^{-\frac{1}{p}} \leq 1$. So, we deduce that $1-|\varphi(z)|^{2}=1$ for all $z \in D$, and this gives $(2)$.

$(2) \Longrightarrow(1)$ For all $f \in H^{p}, C_{\varphi} f=f \circ \varphi \equiv f(0) \in H^{\infty}$. Consequently, $\left\|C_{\varphi} f\right\|_{\infty}=$ $|f(0)| \leq\|f\|_{p}$.

\section{ACKNOWLEDGEMENTS}

This work is part of the author's thesis written under the supervision of Professor H. Queffelec. We would like to thank the referee for his very careful reading and his clever remarks.

\section{REFERENCES}

[1] J. B. Conway, A course in Functional Analysis, Second Edition. Springer-Verlag New York (1990). MR 91e:46001

[2] C. C. Cowen and B. D. MacCluer, Composition Operators on Spaces of Analytic Functions, CRC Press, Boca Raton (1995). MR 97i:47056

[3] A. Denjoy, Sur l'itération des fonctions analytiques, C. R. Acad. Sci. Paris Sér. A., 182 (1926) 255-257.

[4] N. Dunford and J. T. Schwartz, Linear Operators, II (1963). MR 32:6181

[5] P. L. Duren, Theory of $H^{p}$ Spaces, Academic Press (1970). MR 42:3552

[6] G. Hoever, Two classroom proofs concerning composition operators, Integr. Equ. Oper. Theory, 27 (1997) 493-496. MR 98b:47041

[7] H. Hunziker and H. Jarchow, Composition operators which improve integrability, Math. Nachr., 152 (1991) 83-99. MR 93d:47061

[8] N. Jaoua, Propriétés de similarité et de contractivité de certains opérateurs de composition sur des classes de fonctions anlytiques, Thèse, Université de Lille (1999).

[9] H. Kamowitz, The spectra of composition operators on $H^{p}$, J. Funct. Analysis, 18 (1975) 132-150. MR 53:11417

[10] E. Nordgren, Composition operators, Canadian J. Math., 20 (1968) 442-449. MR 36:6961

[11] _ Composition operators on Hilbert spaces, Lecture Notes in Math., 693 (1977), 37-63. MR 80d:47046

[12] G. Pisier, Similarity problems and completely bounded maps, Springer Lecture Notes, 1618 (1996). MR 98d:47002

[13] A polynomially bounded operator on Hilbert space which is not similar to a contraction, J. Amer. Math. Soc., 10, 2 (1997) 351-369. MR 97f:47002

[14] H. J. Schwartz, Composition operators on $H^{p}$, Thesis, University of Toledo (1969). 
[15] F. B. Weissler, Logarithmic Sobolev inequalities and hypercontracive estimates on the circle, J. Funct. Analysis, 37 (1980) 218-234. MR 81k:42007

[16] J. Wolff, Sur l'itération des fonctions analytiques, C. R. Acad. Sci. Paris Sér. A. 182 (1926) $42-43$.

[17] K. Zhu, Operator Theory in Function Spaces, Marcel Dekker, New York, (1990). MR 92c: 47031

Department of Mathematics, Université Lille I, Cité Scientifique, F-59655 Villeneuve D'ASCQ, France

E-mail address: jaoua@agat.univ-lille1.fr 